13,14

\title{
Влияние кислорода на электропроводящие свойства тонких пленок полимерного диэлектрика
}

\author{
(C) А.Р. Юсупов ${ }^{1}$, А.Н. Лачинов ${ }^{1,2}$, Л.Р. Калимуллина ${ }^{1}$, Р.М. Гадиев ${ }^{1}$, Д.В. Никитина ${ }^{1}$ \\ ${ }^{1}$ Башкирский государственный педагогический университет им. М. Акмуллы, \\ Уфра, Россия \\ ${ }^{2}$ Институт фризики молекул и кристаллов УФИЦ РАН, \\ Уфра, Россия \\ E-mail: Azat.yusupov@bk.ru
}

(Поступила в Редакцию 14 июня 2018 г.

В окончательной редакции 15 октября 2018 г.)

Обсуждается влияние атмосферы на электропроводность пленок полидифениленфталида (ПДФ) в структуре типа „сэндвич“ ITO-ПДФ-Sb. На основе электрофизических измерений, а также квантово-химического моделирования делается вывод об основном вкладе кислорода на электропроводность пленок ПДФ.

DOI: 10.21883/FTT.2019.03.47254.165

\section{1. Введение}

Общим свойством всех материалов органической электроники является повышенная чувствительность их свойств к условиям внешней среды. Одним из факторов влияющим на электропроводность полимерных материалов является атмосферный кислород, который на ряду с другими газами может влиять на электронные свойства полимеров [1,2]. В работе [3] показано, что взаимодействие с кислородом подобно допированию, при котором происходит формирование поляронных состояний в области запрещенных энергий, что повышает проводимость материала. Часто подобную чувствительность органических полимеров к окружающим газам стараются использовать. Например, в работе [4] рассматривается имплантация атомов кислорода в полимерный материал для повышения сенсорных свойств устройства. В работе [5] обращается внимание на уникальные возможности электропроводящих полимеров при их использовании в качестве материалов для газовых сенсоров. Однако, как правило, воздействие окружающей атмосферы носит негативный характер, приводя к деструкции органического слоя, к возникновению новых электронных состояний, приводящих к существенному изменению электрофизических свойств. В работе [6] был проведен анализ экспериментальных результатов и теоретических расчетов органических соединений, нашедших применение в электронике с целью систематизации данных о природе ловушечных состояний в органических материалах. Было установлено, что в пленках всех электроактивных полимеров имеется ловушечный уровень с близкой энергией $\sim 3-3.5 \mathrm{eV}$ ниже уровня вакуума. Наличие такого уровня объяснялось результатом взаимодействия с кислородом атмосферы. Было сделано предположение о том, что подобный ловушечный уровень может быть типичным для большинства органических материалов органической элек- троники, содержащих делокализованные $\pi$-сопряженные электроны (MEH-PPV, Р3НТ и т.п.). Однако до сих пор продолжается дискуссия об обоснованности подобного обобщения. Помимо традиционных полисопряженных материалов в качестве электропроводящих или электроактивных материалов часто используются несопряженные полимеры [7]. В тонких пленках таких полимеров наблюдается высокая проводимость и высокая чувствительность электропроводности к малому давлению [8], электрическому полю [9], магнитному полю [10]. В частности, к подобным полимерам относятся полиариленфталиды - материалы с большой шириной запрещенной зоны, хорошо растворимые, хемои термостойкие. Кроме того, они демонстрируют стабильность своих свойств от сверхнизких температур [11] вплоть до $360^{\circ} \mathrm{C}$ и чрезвычайно устойчивы к кислотам и щелочам [12]. Используя высокую химическую стабильность этого полимера, процесс изготовления экспериментальных структур и большая часть опубликованных результатов измерений [7] была получена на открытом воздухе при нормальных условиях без ограничения доступа кислорода к материалу. В связи с этим, актуальным является вопрос о влиянии кислорода, а также различных газов, входящих в состав атмосферы воздуха, на электрофизические параметры тонких пленок несопряженных полимеров и сравнение полученных результатов с выводами работ проведенных на сопряженных структурах [6].

\section{2. Объекты и методы исследования}

В качестве полимерного материала были использованы пленки полидифениленфталида (ПДФ). Экспериментальные образцы представляли пленочные структуры типа „сэндвич“ ITO-ПДФ-Sb (рис. 1), где ITO это смесь окислов индия и олова, $\mathrm{Sb}$ - сурьма. В работе [14] влияние кислорода среды на электро- 




Рис. 1. Диаграмма энергетических уровней электродов и полимера. $E_{1}, E_{2}, E_{3}$ энергии групп ловушек относительно уровня вакуума [13]. $E_{\mathrm{F}}-$ уровень Ферми полимера.

проводящие свойства было обнаружено в структуре ITO-ПДФ-Al, имеющей практически важное значение для электролюминесцентных диодов. Однако в такой структуре сложно учесть влияние окисления алюминия на изменение свойств структуры. В связи с этим в настоящей работе было предложено использовать в качестве металлического электрода сурьму. Работа выхода сурьмы $(4.01 \mathrm{eV})$ наиболее близка по значению к работе выхода алюминия $(4.08 \mathrm{eV})$ [15]. В отличии от алюминиевых электродов, электроды из сурьмы менее требовательны к окружающей атмосфере и устойчивы к окислению при комнатных условиях, что позволяет применять данный материал для проведения измерений на воздухе в течении длительного времени.

Пленки ПДФ формировали методом центрифугирования из раствора полимера (5\%) в циклогексаноне, электроды наносились методом термодиффузионного напыления в вакууме на установке TES 12-18. Толщина получаемых экспериментальных структур контролировалось методами атомно-силовой микроскопии и интерферометрии и составляла порядка $\sim 420 \mathrm{~nm}$. В качестве основного метода исследования был выбран метод, основанный на измерении вольтамперных характеристик. Вольтамперные характеристики (BAX) были получены на измерителе параметров полупроводниковых приборов SourceMeter KEITHLEY 2400. Все измерения проводились в специальной камере с регулируемой газовой атмосферой.

Квантово-химическое моделирование возможного взаимодействия комплексов мономер - ПДФ с атомами газов контрольной группы $\left(\mathrm{O}_{2}, \mathrm{~N}_{2}, \mathrm{Ar}, \mathrm{He}, \mathrm{CO}_{2}\right)$ проводилось методом теории функционала плотности с использованием гибридного функционала B3LYP.

\section{3. Результаты и их обсуждение}

На рис. 2 представлены типичные ВАХ экспериментальной структуры ITO/ПДФ/Sb в зависимости от давления в измерительной камере в интервале от атмосферного до $16.5 \mathrm{kPa}$. Для учета возможного влияния эффекта формовки полимерной пленки в электрическом поле, предварительно на экспериментальных образцах была проведена серия измерений BAX на воздухе при атмосферном давлении. Полученные результаты позволили сделать вывод о том, что эффект формовки не наблюдается, так как форма ВАХ и величины токов от измерения к измерению не меняются в пределах погрешности измерений.

В процессе уменьшения давления ток, протекающий через полимерную пленку, на разных образцах увеличивался в интервале от 3 до 5 раз. Известна сильная зависимость проводимости тонких пленок ПДФ от одноосного давления [16]. Однако, описанная ранее зависимость противоположна наблюдаемой и не может объяснить рост проводимости при уменьшении гидростатического давления. Естественно предположить, что в заданных условиях эксперимента увеличение проводимости при уменьшении давления может быть связано с уменьшением концентрации определенной компоненты воздушной смеси.

На вставке рис. 2 показано изменение тока, протекающего через экспериментальную структуру, от давления при разности потенциалов на электродах $10 \mathrm{~V}$. Анализ полученных результатов показал, что при выдержке образца при минимальном давлении продолжает наблюдаться увеличение проводимости. Однако через 75-80 min изменение проводимости прекращается и ток стабилизируется. Подобное изменение тока связано, повидимому, с дегазацией полимерной пленки от адсорбированных газов из состава атмосферы.

Необходимо отметить, что данный процесс является обратимым: при увеличении давления путем напуска



Рис. 2. ВАХ, измеренные последовательно при уменьшении давления в измерительной камере в процессе ее вакуумирования до $16.5 \mathrm{kPa}(1-100,2-25,3-20,4-17.5$, $5-16.5 \mathrm{kPa})$. На вставке приведена зависимость максимальных токов (при $10 \mathrm{~V})$ от давления. 


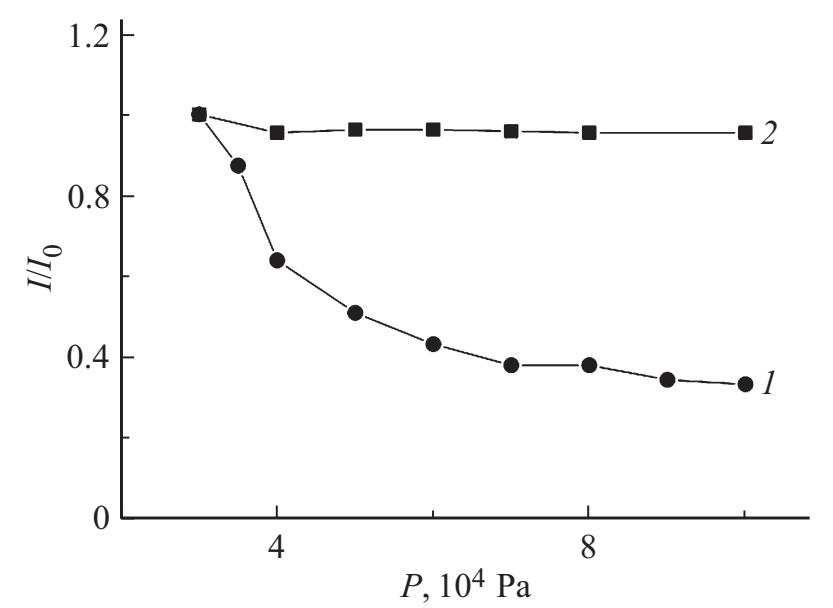

Рис. 3. Зависимость относительного изменения тока от давления при изменении концентрации воздуха (кривая 1) и (кривая 2) для структуры Al/ПДФ/Sb при напряжении $10 \mathrm{~V}$.

воздуха в вакуумную камеру, величина тока уменьшается до первоначального значения (рис. 3, кривая 1). При напуске в камеру инертного газа уменьшения проводимости с ростом давления не наблюдалось. На рис. 3 (кривая 2) это продемонстрировано на примере использования аргона.

Известно, что присутствие молекул воды и кислорода может сильно влиять на электронные свойства структуры металл/полимер/металл [17]. Уместно предположить, что именно кислород, как один из важных компонентов атмосферы (второй по концентрации после азота) оказывает негативное влияние на электропроводимость полимерной пленки. Пары воды были исключены, так как известно их обратное влияние на проводимость полимера: с увеличением концентрации паров проводимость возрастает [18].

Полученные нелинейные ВАХ были проанализированы в рамках теории токов, ограниченных объемным зарядом (ТОО3), модифицированной теорией Пула-Френкеля (ТПФ), подробно описанной в работах $[19,20]$. Согласной этой модели ток, протекающий через тонкую пленку в отсутствии ловушек, описывается хорошо известной формулой:

$$
J=\frac{9}{8} \mu \varepsilon \varepsilon_{0} \frac{V^{2}}{L^{3}},
$$

где $J$ - плотность тока, $\varepsilon-$ относительная диэлектрическая проницаемость (в расчетах принимали $\varepsilon=3.4$ ), $\varepsilon_{0}-$ электрическая постоянная, $\mu-$ эффективная подвижность носителей заряда, $L-$ толщина пленки.

В случае, когда в материале есть ловушки, величина тока зависит от соотношения концентрации свободных носителей заряда $(n)$ к концентрации носителей, захваченных в ловушки $\left(n_{\text {trapped }}\right)-\Theta$. Величина тока в этом случае определяется соотношением:

$$
J=\mu \varepsilon \varepsilon_{0} \Theta_{\text {modern }} \frac{V^{2}}{L^{3}} .
$$

Для учета эффекта Пула-Френкеля в теории токов, ограниченных объемным зарядом, было использовано распределение Ферми-Дирака [20]. В связи с этим параметр $\Theta_{\text {modern }}$ с учетом положения квазиуровня Ферми принимает следующий вид:

$$
\begin{aligned}
\Theta_{\text {modern }} & =\frac{n}{n_{\text {trapped }}} \\
& =\frac{N_{c}}{N_{t}}\left[\exp \left[\frac{E_{\mathrm{FQ}}-E_{C}}{k T}\right]+\exp \left[\frac{E_{t}-E_{C}}{k T}\right]\right],
\end{aligned}
$$

где $N_{c}$ и $N_{t}$ - плотность состояний в зоне проводимости и плотность ловушечных состояний соответственно, $E_{\mathrm{FQ}}-$ квазиуровень Ферми, $E_{C}-$ зона проводимости, $E_{t}$ - энергия равновесного уровня Ферми [20].

Из соотношения (3) следует, что изменение концентрации ловушечных состояний $n_{\text {trapped }}$, например, уменьшение, должно привести к увеличению протекающего тока.

Для проведения анализа полученных данных ВАХ были перестроены в логарифмических координатах и выделены участки линейной аппроксимации (рис. 4). Было установлено, что существует достаточно оснований считать, что при уменьшении давления в рабочей камере (уменьшения концентрации кислорода) происходит снижение концентрации ловушечных состояний в объеме полимерной пленки. В частности, согласно рис. 4 можно заключить, что наблюдается увеличение проводимости образца, увеличение крутизны кривой в области напряжений предельного заполнения ловушек (ПЗЛ), а также происходит смещение режима ПЗЛ в область более слабых полей. Все это наблюдается при таких неизменных условиях, как толщина полимерной пленки, одинаковой равновесной концентрации собственных носителей заряда, работ выхода электрода и полимера и т.д. Кроме того, как показал анализ полученных $\mathrm{BAX}$ (рис. 4) по мере откачки воздуха из вакуумной

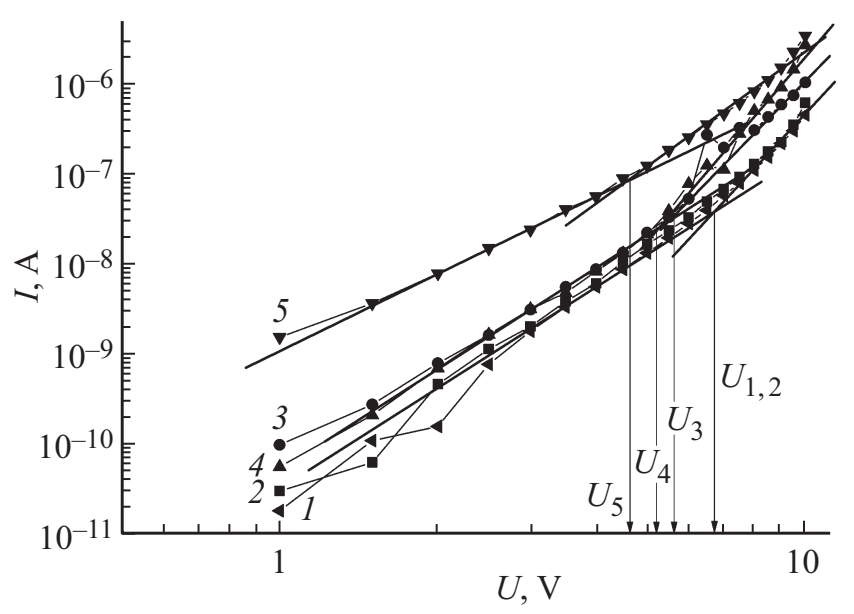

Рис. 4. ВАХ исследуемой структуры при различных значениях давления в вакуумной камере в двойных логарифмических координатах. $(1-100,2-25,3-20,4-17.5,5-16.5 \mathrm{kPa})$. $U_{1}-U_{5}$ напряжения соответствующие точке перегиба. 


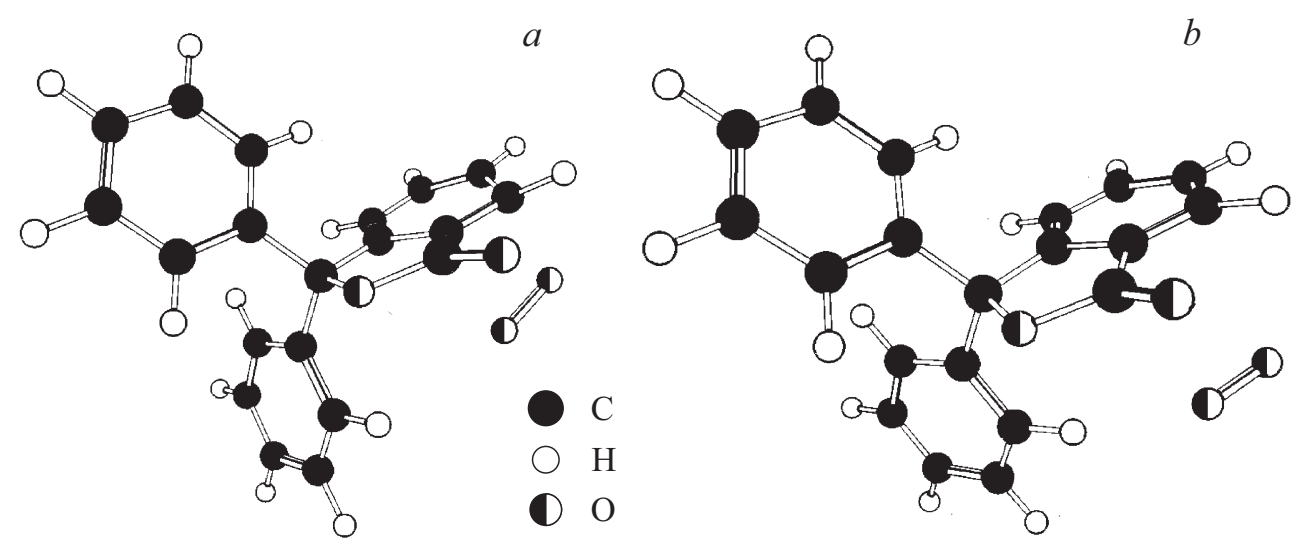

Рис. 5. Трехмерное изображение комплекса ПДФ/О $\mathrm{O}_{2}: a$ до оптимизации, $b$ после оптимизации. $\bigcirc-$ атомы углерода, $\bigcirc-$ водорода, D - кислорода.

камеры изменяется начальный наклон ВАХ. Так при $16.5 \mathrm{kPa}$ в рабочей камере, начальный наклон становится равным 3. В то время как при атмосферном давлении он составляет 3.4. При этом согласно модели ТООЗ такое поведение связывают с условием полного заполнения ловушек либо уменьшения их концентрации [21].

Оценки подвижности носителей заряда, а также концентрации ловушек, проведенные согласно (1)-(3), в зависимости от давления в измерительной камере представлены в табл. 1 .

По мере откачки воздуха (уменьшения давления до $16.5 \mathrm{kPa}$ ) подвижность носителей заряда возрастает в $\sim 8$ раз, что достаточно хорошо согласуется с ранее полученными результатами [22]. В то же время, концентрация ловушечных состояний уменьшается почти на $10 \%$.

Уместно предположить, что уменьшение электропроводности в условиях воздушной атмосферы может быть связано с наличием ловушек, образованных комплексом ПДФ - $\mathrm{O}_{2}$. Наличие диффундировавшего в объем пленки кислорода подтверждается длительным периодом выхода проводимости на стационарный режим. Повидимому, кислород хорошо диффундирует в объем полимерной пленки, создавая дополнительные ловушечные центры. Возможность существования последних можно исследовать, используя соответствующие квантовохимические расчеты совместно с экспериментальными результатами.

Таблица 1. Зависимость подвижности и концентрации носителей заряда в структуре ITO/ПДФ/Sb от давления в вакуумной камере

\begin{tabular}{c|c|c|c}
\hline № & $P, \mathrm{kPa}$ & $\mu, \mathrm{cm}^{2} / \mathrm{V} \cdot \mathrm{s}$ & $n, \mathrm{~cm}^{-3}$ \\
\hline 1 & 100 & $1.1 \cdot 10^{-6}$ & $5.4 \cdot 10^{15}$ \\
2 & 25 & $1.1 \cdot 10^{-6}$ & $5.4 \cdot 10^{15}$ \\
3 & 20 & $1.6 \cdot 10^{-6}$ & $5.2 \cdot 10^{15}$ \\
4 & 17.5 & $1.8 \cdot 10^{-6}$ & $5.1 \cdot 10^{15}$ \\
5 & 16.5 & $8.9 \cdot 10^{-6}$ & $5 \cdot 10^{15}$
\end{tabular}

В модельном эксперименте молекулы газов помещались вблизи фталидного цикла, поскольку ранее проведенные квантово-химические расчеты показали, что именно в области фталидного цикла локализована нижняя вакантная молекулярная орбиталь. Это означает, что вероятность захвата электрона в этой области максимальна. Также при проведении расчета аниона мономера было установлено, что спиновая плотность максимальна в области фталидного цикла.

На рис. 5. представлены трехмерные изображения комплекса мономер- $\mathrm{O}_{2}$ до и после оптимизации. Следует отметить, что оптимизация для всех комплексов проводилась идентично. Молекулы газа отклонялись на различные расстояния для достижения минимальной энергии, в данном случае молекула кислорода удалялась от мономера на $0.5-0.9 \AA$.

Были получены данные о спиновых плотностях для комплексов мономер ПДФ с инертными газами $\mathrm{Ar}, \mathrm{He}$ и с молекулами $\mathrm{CO}_{2}, \mathrm{~N}_{2}, \mathrm{O}_{2}$. Оценка спиновой плотности проходила в комплексах до и после оптимизации. Из результатов следует, что изменения спиновой плотности в комплексах с инертными газами и молекулой $\mathrm{CO}_{2}$ незначительны и максимальны при взаимодействии с кислородом. Можно предположить, что в результате подобного взаимодействия будут формироваться глубокие электронные ловушки, приводящие к уменьшению плотности протекающего тока.

Результаты этих расчетов согласуются с выводами работы [6], где говорится об уменьшении электронной плотности на фрагменте молекулы, взаимодействующем с $\mathrm{O}_{2}$. Большой интерес представляют результаты квантово-химических расчетов энергий сродства к электрону и потенциала ионизации комплексов мономер-ПДФ с молекулами $\mathrm{Ar}, \mathrm{O}_{2}$ и $\mathrm{N}_{2}$ (табл. 2).

Полученные значения энергий свидетельствуют о существенном влиянии кислорода на молекулу мономера. Так сродство к электрону комплекса мономера с кислородом изменяется практически в 2 раза, по сравнению со сродством исходного мономера. В соответствии с 
Таблица 2. Энергии потенциала ионизации и сродства к электрону для комплексов мономер/газ

\begin{tabular}{|c|c|c|c|c|}
\hline \multirow{2}{*}{ Параметр } & \multirow{2}{*}{$\begin{array}{c}\text { Мономер } \\
\text { ПДФ }\end{array}$} & \multicolumn{3}{|c|}{ Комплекс мономер } \\
\hline & & ПДФ/O 2 & ПДФ/Ar & ПДФ/ $\mathrm{N}_{2}$ \\
\hline $\begin{array}{l}\text { Потенциал } \\
\text { ионизации, eV }\end{array}$ & 6.9 & 6.3 & 6.9 & 6.9 \\
\hline $\begin{array}{l}\text { Сродство } \\
\text { к электрону, } \mathrm{eV}\end{array}$ & 1.7 & 4.3 & 1.7 & 1.7 \\
\hline
\end{tabular}

моделью Свораковского [22], наличие примесных состояний с энергией сродства к электрону большего, чем в основном материале, приводит к формированию электронных ловушек глубиной:

$$
\Delta E=\xi_{G}-\xi_{H}+E_{P}
$$

где $\xi_{G}-$ энергия электронного сродства примеси (гость), $\xi_{H}$ - энергия электронного сродства основного материала (хозяин), $E_{P}$ - энергия поляризации среды.

Можно сделать вывод о том, что результаты проведенных квантово-химических расчетов говорят о влиянии $\mathrm{O}_{2}$ на ПДФ и свидетельствуют о возможном возникновении в полимерном материале глубоких кислородных ловушек. Как и в работе [6] подобные ловушки понижают подвижность носителей заряда и уменьшают проводимость материала.

Аналогичные расчеты были проведены для комплексов с азотом и аргоном и углекислым газом, которые показали либо отсутствие, либо значительно более слабое по сравнению с кислородом взаимодействие с молекулой полимера.

В работе [6] изменение энергий комплексов $\mathrm{PPV} / \mathrm{H}_{2} \mathrm{O}$ связывают с образованием продуктов окисления, которые играют роль ловушек и понижают подвижность носителей заряда. Представляет большой интерес диаграмма, на которой авторы [6] показали результаты измерений нижних вакантных молекулярных орбиталей $(\mathrm{HBMO})$ и энергию ловушек для 6 наиболее известных полимеров: NRS-PPV, $\mathrm{OC}_{1} \mathrm{C}_{10}-\mathrm{PPV}, \mathrm{P} 3 \mathrm{HT}, \mathrm{F} 8 \mathrm{BT}$,



Рис. 6. Схематичное изображение энергий НВМО и центр распределения ловушек. Сплошные линии обозначают НВМО уровни, пунктирные линии показывают ловушечные уровни.
PF10TBT, РCPDTBT (рис. 6). Анализ представленных данных позволил авторам установить схожее для разных соединений распределение электронных ловушек с глубиной залегания на $3.6 \mathrm{eV}$ ниже уровня вакуума, с типичной шириной распределения $0.1 \mathrm{eV}$ и концентрацией ловушек $\sim 3 \cdot 10^{17} \mathrm{~cm}^{-3}$. В отличие от соотношения (4) в работе [1] отсчет энергий электронных состояний проводили относительно уровня вакуума.

Мы дополнили эту диаграмму результатами для полидифениленфталида (крайняя левая позиция на диаграмме рис. 6). Можно видеть, что эти результаты не противоречат выводам, сделанным в работе [6].

Ранее [6] было сделано предположение, согласно которому электронные ловушки имеют общую природу, обусловленную образованием комплексов гидратированного кислорода. Следствием этого факта является то, что ловушка, ограничивающая электронный ток, может быть предсказана для любого полимера. Согласно результатам исследования тонких полимерных пленок ПДФ [13] методами термостимулированных токов и вольтамперных характеристик, в запрещенной зоне полимера можно выделить три группы электронных ловушек с максимумами плотности состояния при энергиях $0.5,1.06$ и $2.4 \mathrm{eV}$ относительно дна зоны проводимости. Следовательно, можно говорить о нахождении ловушки с энергией $3.06 \mathrm{eV}$ ниже уровня вакуума для ПДФ, которая определяется наличием кислорода в объеме полимера [13].

\section{4. Заключение}

Таким образом, установлено, что наличие кислорода приводит к уменьшению проводимости пленок полидифениленфталида за счет формирования ловушек носителей заряда. Причем при взаимодействии кислорода c фрагментами полимерной молекулы не возникает химической связи, что приводит к обратимому изменению электропроводности при изменении концентрации кислорода. Благодаря этому пленки ПДФ демонстрируют высокую стабильность электрофизических характеристик в течение нескольких лет при проведении повторных измерений в условиях открытой атмосферы и при комнатной температуре.

Авторы выражают благодарность д.Х.Н. профессору С.Н. Салазкину за помощь в получении объекта исследования.

\section{Список литературы}

[1] N.T. Kemp, G.U. Fianagan, A.B. Kaiser, H.J. Trodahl, B. Chapmana, A.C. Partridge, R.G. Buckley. Synthetic Met. 101, 1-3, 434 (1999).

[2] E. Vitoratos, S. Sakkopoulos, N. Paliatsas, K. Emmanouil, S.A. Choulis. J. Organic Polymer Mater. 2, 7 (2012).

[3] S.K. Singh, X. Crispin, I.V. Zozoulenko. J. Phys. Chem. C 121, 22, 12270 (2017). 
[4] K.V. Aneesh Kumar, G.N. Kumaraswamy, C. Ranganathaiah, H.B. Ravikumar. J. Appl. Polymer. Sci. 134, 44962 (2017).

[5] Hua Bai, Gaoquan Shi. Sensors 7, 267 (2007).

[6] T. Nicolai, M.G. Kuik, A.H. Wetzelaer, B. Boer., C. Campbell, C. Risko, P.W.M. Blom. Nature Mater, 11, 882 (2012).

[7] А.Н. Лачинов, Н.В. Воробьева. УФН 176, 12 (2006).

[8] А.Н. Лачинов, А.Ю. Жеребов, В.М. Корнилов. Письма в ЖЭТФ 52, 2, 742 (1990).

[9] A.Yu. Zherebov, A.N. Lachinov. Synth. Met. 44, 99 (1991).

[10] Н.В. Воробьева, А.Н. Лачинов, Б.А. Логинов. Поверхность: Рентген., синхротрон. и нейтрон. исследование 5,22 (2006).

[11] A.N. Ionov, A.N. Lachinov, M.M. Rivkin, V.M. Tuchkevich. Solid State Commun 82, 8, 609 (1992).

[12] С.Н. Салазкин. Высокомолекуляр. соединения 46, 7, 1244 (2004).

[13] А.С. Накаряков, А.Н. Лачинов, А.Ф. Пономарев, Е.Е. Цеплин, В.А. Антипин. ФТТ 53, 12, 2397 (2011).

[14] А.Р. Юсупов, А.Р. Тамеев, А.Н. Лачинов, В.С. Любцов, А.В. Ванников. Письма в ЖТФ 38, 24 (2012).

[15] A.M. Eugene. Properties of Materials. In: Reference Data For Engineers: Radio, Electronics, Computer and Communications / Eds Mac E. Van Valkenburg, Wendy M. Middleton. Woburn, MA: Butterworth-Heinemann (2002).

[16] A.N. Lachinov, A.Yu. Zherebov, V.M. Kornilov. Synth. Met. 44, 111 (1991)

[17] S.A. Abdou Mohamed, P. Orfino Francesco, S. Yongkeun, S. Holdcroft. J. Am. Chem. Soc. 119, 4518 (1997).

[18] Р.Б. Салихов, А.Н. Лачинов, Р.Г. Рахмеев, Р.М. Гадиев, А.Р. Юсупов, С.Н. Салазкин. Измерительная техника 4, 62 (2009).

[19] P.N. Murgatroyd. J. Phys. D 3, 51 (1970).

[20] D.F. Barbe. J. Phys. D 4, 1812 (1971).

[21] Ф. Гутман, Л. Лайонс. Органические полупроводники / Под ред. Е.Л. Франкевича. Мир, М. (1970). С. 696.

[22] J. Sworakowski. Mol. Cryst. Liq. Cryst. 11, 1 (1970).

Редактор Д.В. Жуманов 